\title{
Feasibility study of Lense-Thirring precession in LS I $+61^{\circ} 303$
}

\author{
M. Massi and L. Zimmermann
}

\author{
Max Planck Institut für Radioastronomie, Auf dem Hügel 69, 53121 Bonn, Germany \\ e-mail: [mmassi;lzimmerm] @mpifr-bonn.mpg.de
}

Received 2 December 2009 / Accepted 18 March 2010

\begin{abstract}
Context. Very recent analysis of the radio spectral index and high energy observations have shown that the two-peak accretion/ejection microquasar model applies for LS I $+61^{\circ} 303$.

Aims. The fast variations of the position angle observed with MERLIN and confirmed by consecutive VLBA images must therefore be explained in the context of the microquasar scenario.

Methods. We calculate what could be the precessional period for the accretion disk in LS $\mathrm{I}+61^{\circ} 303$ under tidal forces of the Be star $\left(P_{\text {tidal-forces }}\right)$ or under the effect of frame dragging produced by the rotation of the compact object $\left(P_{\text {Lense-Thirring }}\right)$.

Results. $P_{\text {tidal-forces }}$ is more than one year. $P_{\text {Lense-Thirring }}$ depends on the truncated radius of the accretion disk, $R_{\mathrm{tr}}$. We determined $R_{\mathrm{tr}}=300 r_{\mathrm{g}}$ for observed QPO at $2 \mathrm{~Hz}$. This value is much above the few $r_{\mathrm{g}}$, where the Bardeen-Petterson effect should align the midplane of the disk. For this truncated radius of the accretion disk $P_{\text {Lense-Thirring }}$ for a slow rotator results in a few days.

Conclusions. Lense-Thirring precession induced by a slowly rotating compact object could be compatible with the daily variations of the ejecta angle observed in LS I $+61^{\circ} 303$.
\end{abstract}

Key words. black hole physics - radio continuum: stars - pulsars: general - X-rays: binaries $-\mathrm{X}$-rays: indiviual $\left(\mathrm{LS}\right.$ I $\left.+61^{\circ} 303\right)$

\section{Introduction}

LS I $+61^{\circ} 303$ is a X-ray binary formed by a compact object and a massive star with an optical spectrum typical for a rapidly rotating B0 V star (Hutchings \& Crampton 1981). The Be star with a fast polar wind has an equatorial dense, low velocity wind with a power law density distribution of the form $\rho_{\mathrm{w}}(R)=\rho_{0}\left(R / R_{*}\right)^{-3}$, where $R_{*}$ is the radius of the primary Be star (Waters et al. 1988; Martí \& Paredes 1995). The real nature of the compact object travelling in this stratified wind on an eccentric orbit with $e=0.54-0.7$ is still unknown (Aragona et al. 2009; Casares et al. 2005). In fact, because of the uncertainty in the inclination of the orbit $i=30^{\circ} \pm 20^{\circ}$, the compact object could be either a neutron star or a black hole of 3-4 $M_{\odot}$. Two radio periodicities are present (Gregory 2002), one of $26.5 \mathrm{~d}$ (phase $\Phi$ ), which corresponds to the orbital period, and a second one of $4.6 \mathrm{yr}$ (phase $\Theta$ ) which is related to variations in the equatorial wind of the Be star (Zamanov \& Marti 2000). In the past two scenarios were presented for the system. One is that the compact object is a young, still very fast rotating, strongly magnetized pulsar, whose relativistic wind collides with the Be star's wind and prevents any accretion. In this scenario the prolonged strong wind interaction during the periastron passage should continuously accelerate particles to relativistic velocities and a prolonged optically thin outburst is expected, as it occurs in the young pulsar PSR B1259-63 (Fig. 3 of Connors et al. 2002). PSR B1259-63, which clearly is a pulsar, as seen by its pulses with a period of $48 \mathrm{~ms}$ observed by Johnston et al. (1992), shows a large optically thin radio outburst around the periastron passage. The other scenario for LS I $+61^{\circ} 303$ is that the compact object is an accreting black hole or a low magnetic field neutron star. From Bondi (1952) the wind accretion rate is proportional to $\frac{\rho_{\mathrm{w}}}{v^{3}}$, i.e. directly proportional to the wind density and inversely proportional to the cubic of the relative speed between the compact object and the
Be wind. In an eccentric orbit this different relationship for density and velocity creates two peaks in the accretion rate curve, one at periastron where the density is at its maximum and a second one when the drop in density is compensated by the decrease in velocity towards apastron. Taylor et al. (1992) computed the accretion rate curve for different eccentricities and showed that two peaks begin to appear for an eccentricity above 0.4 . Whereas the first peak is always toward periastron, the orbital occurrence of the second accretion peak depends on variations of the wind of the Be star. Marti \& Paredes (1995) computed the accretion rate curve for different wind velocities, associated with the variability of the Be star, and showed that for a stellar wind velocity of $20 \mathrm{~km} \mathrm{~s}^{-1}$ the two peaks become rather close to each other, whereas for a wind velocity of $5 \mathrm{~km} \mathrm{~s}^{-1}$ they are at their maximum orbital offset of $\Delta \Phi=0.4$. This value, for an orbital period $P_{\text {orbital }}=26.5 \mathrm{~d}$, corresponds to almost 11 days, i.e. the second peak may occur almost at apastron. Bosh-Ramon et al. (2006) showed that around periastron $(\Phi=0.23)$ only a small radio outburst is expected in coincidence with the first accretion peak. That occurs, because of the severe external inverse Compton (EIC) losses of the electrons, which upscatter UV stellar photons of the close Be star to high energies. In other words, associated with the first accretion/ejection peak a high energy outburst due to EIC is expected along with a small radio outburst. The second displaced accretion/ejection peak, instead, should be observed in the radio band as a large outburst. In particular this large radio outburst, $S \propto v^{\alpha}$, should follow the characteristics of microquasars: optically thick emission, i.e. $\alpha \geq 0$, followed by an optically thin outburst, i.e. $\alpha<0$ (Fender et al. 2004). The first type of emission, the optically thick radio emission, is related in microquasars to a steady, low velocity jet centered on the orbit. The following optically thin outburst is related to a transient jet, associated with shocks quite displaced from the center (see Fig. 1). With this transient jet very high energy 
Table 1. Two-peak MQ vs Pulsar Model.

\begin{tabular}{|c|c|c|c|}
\hline & PSR B1259-63 & Two-peak Microquasar & 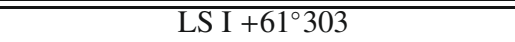 \\
\hline radiopulses & yes & no & no \\
\hline$L_{\mathrm{X}}[\mathrm{erg} / \mathrm{s}]$ & $5 \times 10^{34 a}$ & $10^{33.5}-10^{36 b}$ & $(1-6) \times 10^{34 c}$ \\
\hline Largest radio outburst & toward periastron $^{d}$ & toward apastron ${ }^{e}$ & toward apastron ${ }^{f}$ \\
\hline Radio spectral index & $\alpha<0^{d}$ & $\alpha \geq 0$ then $\alpha<0^{g}$ & $\alpha \geq 0$ then $\alpha<0$ twice per orbit ${ }^{b}$ \\
\hline Gamma-ray peak & toward periastron $^{h}$ & EIC at periastron, dominant SSC toward apastron ${ }^{e, i}$ & Two peaks: EGRET/VERITAS/FERMI ${ }^{i}$ \\
\hline
\end{tabular}

Notes. ${ }^{(a)}$ Kaspi et al. 1995. ${ }^{(b)}$ Massi \& Kaufman Bernadó 2009. ${ }^{(c)}$ Paredes et al. 1997. ${ }^{(d)}$ Connors et al. 2002. ${ }^{(e)}$ Bosh-Ramon 2006. ${ }^{(f)}$ Fig. 2-c in Massi \& Kaufman Bernadó 2009. ${ }^{(g)}$ Fender et al. 2004. ${ }^{(h)}$ Aharonian et al. 2009. ${ }^{(i)}$ see Sect. 1.

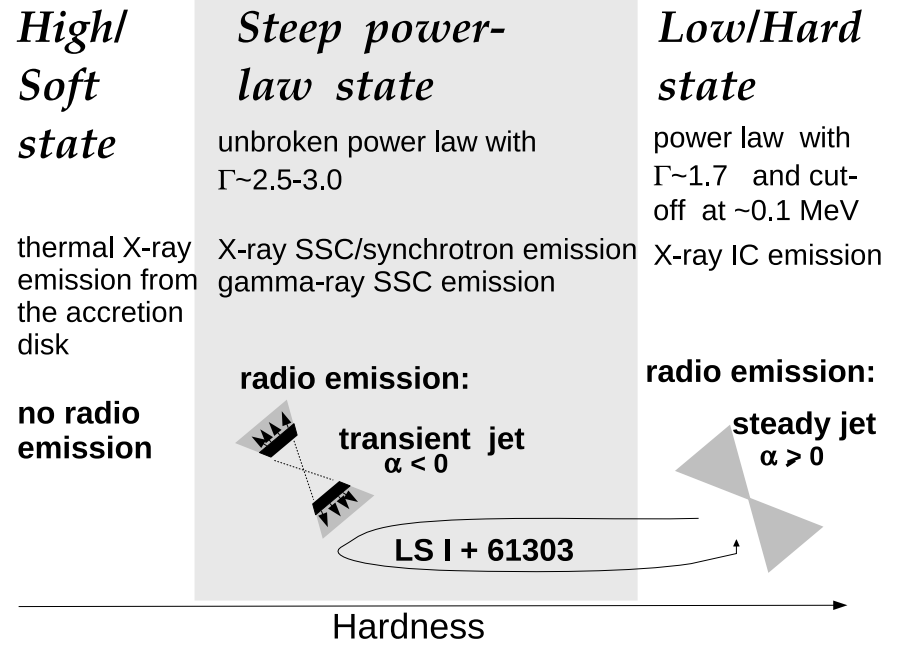

Fig. 1. X-ray states/radio states vs. hardness. Along with the usual $\mathrm{X}$-ray characteristics (McClintock \& Remillard 2006) and radio characteristics (Fender et al. 2004) of the spectral states, we add for the Steep power-law state also the X-and gamma-ray characteristics for the shock-in-jet model and for blazars (Marscher \& Gear 1985; Katarzyński et al. 2005; Katarzyński \& Walczewska 2010). The transient radio jet, i.e. "plasmoids" displaced from the center, is related to shocks travelling in the slowly moving preexisting steady jet established during the previous low hard state. LS I $+61^{\circ} 303$ seems to evolve from a very low low/hard state to a steep power-law state. Then it develops again a steady jet centered on the system (i.e. low/hard state) and never reaches the thermal high/soft state.

emission is expected as well, because of dominating self synchrotron Compton (SSC) losses during the growing phase of the shock (Marscher \& Gear 1985).

How does LS I $+61^{\circ} 303$ fit in the two different scenarios? As a matter of fact, radio pulses have never been observed in LS I $+61^{\circ} 303$, moreover the large outburst in LS I $+61^{\circ} 303$ is clearly shifted towards apastron contrary to the case of PSR B1259-63 (Table 1). In addition, the recent analysis of the radio spectral index, $\alpha$, by Massi \& Kaufman Bernadó (2009) show the clear sequence typical for microquasars: optically thick emission (steady jet) and optically thin emission (transient jet). The quite impressive fact in $\mathrm{LS} \mathrm{I}+61^{\circ} 303$ is that during the maximum of the $4.6 \mathrm{yr}$ periodicity, i.e $\Theta=0.7-1.3$, this sequence (optically thick emission / optically thin emission) occurs twice along the orbit of 26.5 d. In Fig. 3 in Massi \& Kaufman Bernadó (2009) one sees how even the small radio peak at periastron, attenuated because of severe EIC losses and nearly negligible in terms of flux density in comparison to the large $\Delta \Phi=0.3$ outburst, presents the same very clear variation in the spectral index, from $\alpha \geq 0$ (steady jet) to $\alpha<0$ (transient jet), which proves the two-peak accretion model in the radio band.
In terms of high energy emission as discussed above two peaks are predicted, each of them formed by different EIC and SSC contributions. At periastron one expects dominant EIC contribution to the gamma-ray emission, due to the proximity of the Be star. Towards apastron one expects the contrary: Strong gamma-ray emission due to SSC associated with the powerful transient jet with additional EIC gamma-ray emission that depends on the distance from the Be star. Two emission peaks are confirmed by observations at high energies. One data set of EGRET shows evidence of the periastron peak. A second data set shows a hint of a peak at periastron and a second peak shifted towards apastron $(\Phi=0.5)$ (Massi et al. 2005). The recent Fermi LAT observations confirm the two peaks (Abdo et al. 2009). The Fermi light curve is characterized by a broad peak after periastron as well as a smaller peak just before apastron. TeV observations with VERITAS (Acciari et al. 2009) along with the strong emission at $\Phi=0.5-0.9$, first detected with MAGIC (Albert et al. 2006), also give marginal evidence for emission at $\Phi=0.2-0.3$.

LS I $+61^{\circ} 303$ fits then well the two-peak microquasar scenario. Its radio properties show a recurrent switch between transient jet and steady jet, the two radio states. As discussed in Fender et al. (2004) in microquasars the two radio states, steady and transient jet, are simultaneous with two X-ray states: the low/hard state and the steep power law state respectively (shown in Fig. 1). This seems to happen also in LS I +61 303. As analysed in Massi \& Kaufman Bernadó (2009) INTEGRAL (keV-MeV) observations by Chernyakova et al. (2006) with the typical photon index $\Gamma=1.4-1.8$ of the low/hard state occur at $\Theta$ and $\Phi$ where optically thick radio emission is observed, i.e. $\alpha \geq 0$. INTEGRAL observations with $\Gamma=3.6_{-1.1}^{+1.6}$, are measured at $\Theta$ and $\Phi$ where radio emission with $\alpha<0$ is observed. Moreover, when $\mathrm{TeV}$ emission is detected in $\mathrm{LS} \mathrm{I}+61^{\circ} 303$ with Cherenkov telescopes the energy spectrum is always well fitted by a power law with a photon index $\Gamma \simeq 2.6$ (Albert et al. 2009; Acciari et al. 2009) independently of changes in the flux level as expected for the steep power law state, where the photon index is a fundamental property of the state and not the luminosity (see discussion in Massi \& Kaufman Bernadó 2009). Of particular interest are the recent observations by Anderhub et al. (2009) probing correlated X-ray emission, attributed to synchrotron radiation, and VHE emission. We note that the corrected X-ray flux of $9 \times 10^{-12} \mathrm{erg} \mathrm{cm}^{-2} \mathrm{~s}^{-1}$ and the VHE flux of $11 \times 10^{-12} \mathrm{erg} \mathrm{cm}^{-2} \mathrm{~s}^{-1}$ result in $F_{\mathrm{VHE}} \propto F_{\mathrm{X}}^{\eta}$ with $\eta=0.99$, in agreement with the correlation observed in blazars, where $\eta$ is in the range 0.99-3 (Katarzyński \& Walczewska 2010). The high energy spectra of $\mathrm{TeV}$ blazars are explained with a model where relativistic electrons accelerated in a shock emit synchrotron radiation up to X-rays. A fraction of this emission is upscattered to higher energies by the same population of the electrons (SSC) (Katarzyński et al. 2005). This agrees with the predictions of the shock-in-jet model invoked for the optically thin radio outburst 
of the transient jet described above and associated to the steep power-law state. Because of the correspondence between X-ray states and radio states we suggest that the recurrent switch between transient jet and steady jet observed in LS I $+61^{\circ} 303$ corresponds to a continuous switch between a low/hard state and a steep power-law state (Fig. 1). The source is therefore pratically frozen in a permanent microquasar state, in fact it is always radio loud, never reaching, as other X-ray binaries the high/soft thermal state.

The peculiar aspect of LS I $+61^{\circ} 303$, that we will deal with in this study, is its short-term variability. Variability is the main characteristic of blazars and micro-blazars, where the shock is seen almost face-on and the Doppler factor is large. LS I $+61^{\circ} 303$ was indicated since 2002 by Kaufman Bernadó et al. as a microblazar, because of its one-sided jet radio morphology, typical of blazars, where the receding jet is Doopler deboosted whereas the flux density of the approching jet is strongly amplified.

LS I $+61^{\circ} 303$ shows strong morphological changes: or the position angle of the one-sided jet continuosly changes, or at some epochs the receding jet appears unattenuated (compare the map of Taylor et al. 2000 with that of Dhawan et al. 2006 in Fig. 1 by Massi \& Kaufman Bernadó 2009). Precession of the accretion disk (and therefore of the jet) causing the jet to point closer to or farther away from the line of sight would explain variable Doppler boosting and variations in the position angle. Precessing jets are well known in microquasars, the most spectacular one being that of SS433, the first microquasar (Dubner et al. 1998). However, in LS $I+61^{\circ} 303$ the variations seem to be very rapid compared to the 164-day precessional period of SS433. The peculiarity of the variations of LS I $+61^{\circ} 303$ is their short timescale. MERLIN images revealed a surprising variation of $60^{\circ}$ in position angle in only one day (Massi et al. 2004). Dhawan et al. (2006) measured in VLBA images a rotation of the inner structure of roughly $5^{\circ}-7^{\circ}$ in $2.5 \mathrm{~h}$, that is again almost $60^{\circ}$ day. Indeed, because of the difficulty to explain this puzzling variations in the context of microquasars the variations were interpreted as due to a cometary tail of a pulsar. Now, that the radio spectral index analysis and very high energy observations confirm the two-peak microquasar scenario (see Table 1), an investigation of the physical processes behind these fast variations is necessary. This is the aim of this work.

The most likely cause for precession of an accretion disk of a compact object is an assymetric supernova explosion of the progenitor. As a result the compact object could be tilted (Fragile et al. 2007). In this case either the accretion disk is coplanar with the compact object, and therefore subject to the gravitational torque of the Be star or instead, the accretion disk is coplanar with the orbit, but tilted in respect to the compact object, which induces Lense-Thirring precession if the compact object rotates. In this paper we therefore examine these two possibilities with the aim to quantify them and to compare them with the observed short time scale of variations in LS I $+61^{\circ} 303$.

\section{Tidal Forces in a binary system}

If the accretion disk is tilted with respect to the binary orbital plane, then its precession can be tidally induced by the companion star. The expression for the precession period depends, for a given orbital period $(P=26.496 \mathrm{~d})$ and semi-major axis $\left(a \simeq 10^{12} \mathrm{~cm}\right)$, on the mass ratio $\mu=\frac{M_{*}}{M_{\text {compact object }}}$, the orbital

\section{Tidally forced precession for assumed disk size}

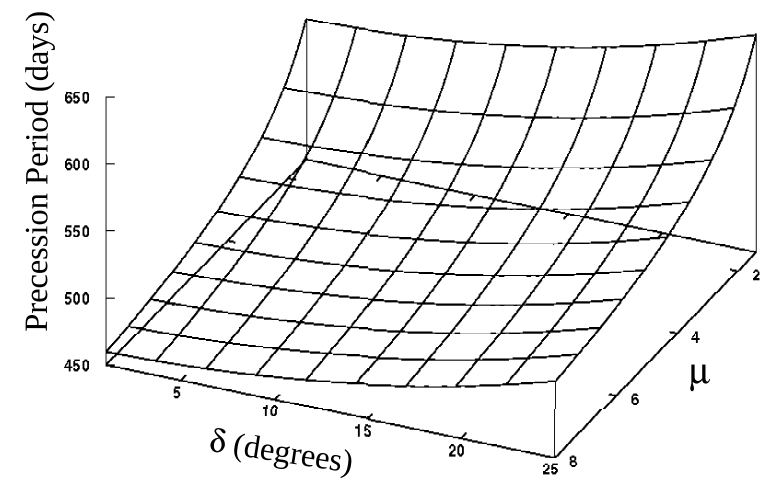

Fig. 2. Precession due to tidal torque induced by the Be star as function of mass ratio, $\mu$, and inclination angle, $\delta$, of the orbital plane with respect to the plane of the disk (Eq. (2)).

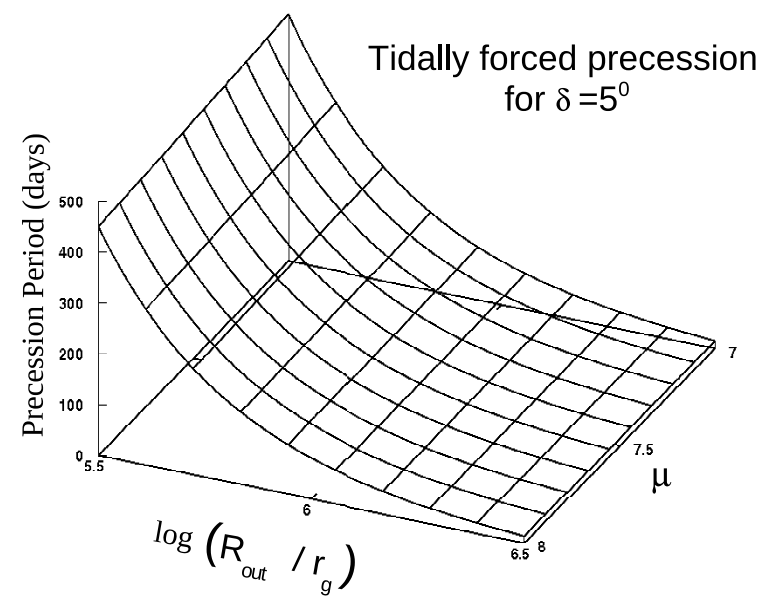

Fig. 3. Precession due to tidal torque induced by the Be star as function of mass ratio, $\mu$, and disk size for inclination angle $\delta=5^{\mathrm{deg}}$ (Eq. (1)).

inclination $\delta$ with respect to the disk and the accretion disk size $R_{\text {out }}$, (Larwood 1998, Eq. (4)):

$P_{\mathrm{TF}}=\frac{61.8}{\cos \delta} \frac{(1+\mu)^{1 / 2}}{\mu}\left(\frac{a}{R_{\mathrm{out}}}\right)^{3 / 2}$

The size of the disk and the orbit can be eliminated, following Larwood (1998), by writing the accretion disc as a fraction $\beta$ of the Roche lobe. Then both $\beta$ and the Roche lobe can be written as a function of $\mu$, and Eq. (1) then becomes:

$P_{\mathrm{TF}}=\frac{61.8}{\cos \delta} \frac{(1+\mu)^{1 / 2}}{\mu}\left[\frac{0.6+\mu^{2 / 3} \ln \left(1+\mu^{-1 / 3}\right)}{0.49 \frac{1.4}{1+[\ln (1.8 \mu)]^{0.24}}}\right]^{3 / 2}$

In LS I $+61^{\circ} 303$ the mass of the compact object is in the range 2-3.5 $M_{\odot}$ and for the Be star one can assume the range 5-15 $M_{\odot}$, with a resulting $\mu$ in the range 1.4-8. In Fig. 2 we plot the precessional period, $P_{\mathrm{TF}}$, as a function of $\delta$ and $\mu$. We see that even for the smallest values of $\delta$ and the highest values of $\mu$ we already get $P_{\mathrm{TF}}=460 \mathrm{~d}$, which is more than one order of magnitude above the orbital period of LS I $+61^{\circ} 303$. This result of a period ratio $\frac{P_{\mathrm{TF}}}{P_{\text {orbit }}} \simeq 17$ agrees well with the expectations of the timescale for a tidally forced precession. In fact, in Table 1 of Larwood (1998) one sees that the period ratio for known precessing X-ray 
binaries lies within the range 8-22. However, such a long precessional period prevents tidal forces to be responsible for the fast variations of the position angle present in the MERLIN observations of Massi et al. (2004) and in the VLBA images of Dhawan et al. (2006).

In our above calculations we assumed a single planar disk. If, instead, only a portion of the disk is warped out of the binary plane, as could be likely (Foulkes et al. 2006), a warp at smaller radii would decrease $R_{\text {out }}$ in Eq. (1) and further increase $P_{\mathrm{TF}}$. The only way to get a smaller $P_{\mathrm{TF}}$ is therefore to increase $R_{\text {out }}$. In Fig. 3 we plot Eq. (1) as a function of $\mu$ and $R_{\text {out }}$ for $\delta=5^{\circ}$. The first interesting result is that the before determined value of $P_{\mathrm{TF}} \simeq 450 \mathrm{~d}$ by using Larwood's (1998) fit for $\beta$, which is derived from Paczyǹski's (1977) values, corresponds to an already very large disk size, $\frac{R_{\text {out }}}{r_{\mathrm{g}}}=10^{5.5}$ (see Fig. 3), where $r_{\mathrm{g}}$ is the gravitational radius, $r_{\mathrm{g}}=M_{\text {compact object }} G / c^{2}$. Indeed, Paczyǹski (1977) argues that his values constitute upper limits to the disk size provided that pressure is sufficiently small in the disk. One could therefore assume larger disks because of higher values of pressure. On the other hand, if we assume a larger disk size, we get the second interesting result that one needs to enlarge the disk size one order of magnitude, reaching the value of $\frac{R_{\text {out }}}{r_{\mathrm{g}}}=10^{6.5}$, to finally reduce $P_{\mathrm{TF}}$ to a few days. Such a large $R_{\text {out }}$ value is unlikely. Already beyond about $10^{4}$ gravitational radii, self-gravitation is larger than central gravitation, and the disk becomes gravitationally unstable (Collin \& Huré 1999).

\section{The lense-thirring effect}

Whenever a spinning compact object has a misaligned accretion disk, the Lense-Thirring precession effect will arise with an angular frequency, $\Omega_{\mathrm{LT}}$, given by (Eq. (29) of Caproni et al. 2006; Wilkins 1972):

$\Omega_{\mathrm{LT}}=\frac{2 \pi}{P_{\mathrm{LT}}}=\frac{2 G J}{c^{2} r^{3}}$

where $J$ is the angular momentum of the compact object and $r$ the radial distance from the rotating compact object to the accretion disk. The angular momentum, $J$, can be expressed in terms of the dimensionless spin parameter, $a_{*}$, such that $J=\frac{a_{*} G M_{\text {compact object }}^{2}}{c}$ (Fragile et al. 2001), and the precessional period (in days) becomes

$P_{\mathrm{LT}}=\frac{1.8 \times 10^{-10}}{a_{*}} \frac{M_{\text {compact object }}}{M_{\odot}}\left(\frac{r}{r_{\mathrm{g}}}\right)^{3}$.

In general, the Bardeen-Petterson effect predicts an alignment of the inner accretion disk with the symmetry plane of the compact object. Nelson \& Papaloizou (2000) showed that the alignment by the Bardeen-Petterson effect extends only to a few gravitational radii (15-30 $r_{\mathrm{g}}$ ) from the compact object. The rest of the disk remains tilted and therefore under the influence of the Lense-Thirring precession effect (Nelson \& Papaloizou 2000). The models of the low/hard state give a geometrically thin, optically thick accretion disk truncated at $R_{\mathrm{tr}} \simeq 100 r_{\mathrm{g}}$ (McClintock \& Remillard 2006) and therefore well above the limit of a few $r_{\mathrm{g}}$. In addition, for LS I $+61^{\circ} 303$ the truncated radius $R_{\text {tr }}$ is expected to be even larger than $r \simeq 100 r_{\mathrm{g}}$ because its state with $L_{\mathrm{X}} \simeq(1-6) \times 10^{34}$ (Table 1) corresponds to a very low low/hard state just above the upper limit of a quiescent state ( $L_{X}$ (quiescent state): $10^{30.5}-10^{33.5} \mathrm{erg} / \mathrm{s}$ McClintock \& Remillard 2006).
Following Stella \& Vietri (1998) we can argue that matter inhomogeneities present at an inner disk boundary cause quasiperiodic oscillations (QPO) at the Keplerian frequency $v_{\mathrm{K}}$. We can estimate the value for $R_{\mathrm{tr}}$ in $\mathrm{LS} \mathrm{I}+61^{\circ} 303$ from observed QPO. During their monitoring with RXTE (2-10 keV) Ray \& Hartman (2008) observed a period of strong variability with a spectrum best fit by a powerlaw of photon index about 1.5 (i.e. low/hard state); a power spectral analysis revealed QPO at $2 \mathrm{~Hz}$. The relativistic Keplerian frequency (Eq. (2) in Caproni et al. 2006) is:

$v_{\mathrm{K}}=\frac{1}{2 \pi} \frac{c}{r_{\mathrm{g}}}\left[\left(\frac{R_{\mathrm{tr}}}{r_{\mathrm{g}}}\right)^{3 / 2}+a_{*}\right]^{-1}$

By solving for $\frac{R_{\mathrm{tr}}}{r_{\mathrm{g}}}$ one determines:

$\frac{R_{\mathrm{tr}}}{r_{\mathrm{g}}}=\left[\frac{32 \times 10^{3}}{\frac{M_{\text {compact object }}}{M_{\odot}} v_{K}}-a_{*}\right]^{2 / 3}$.

Equation (6) for $M_{\text {compact object }}=3 M_{\odot}$ and $v_{K}=2 \mathrm{~Hz}$ clearly reduces to the Newtonian value for any value of $a_{*}$, and therefore to: $\frac{R_{\mathrm{tr}}}{r_{\mathrm{g}}}=300$.

Above, to determine the truncated radius of the accretion disk we set the QPO frequency of $2 \mathrm{~Hz}$ equal to the Keplerian frequency. Below, we analyse if the resulting $\frac{R_{\mathrm{tr}}}{r_{\mathrm{g}}}=300$ could correspond to a precessional period of a few days. This is therefore, different from Ingram et al. (2009) and Ingram \& Done (2010), who associate the low frequency QPO directly to LenseThirring precession. As a matter of fact a truncated radius, $R_{\mathrm{tr}}$, a factor 3 above the value of $100 r_{\mathrm{g}}$ determined for X-ray binaries in low/hard states, is very consistent with the X-ray luminosity value of $\mathrm{LS} \mathrm{I}+61^{\circ} 303$, which indicates a very low low/hard state. Moreover, the escape velocity $v / c=\sqrt{\frac{2 r_{g}}{r}}$ for $r=300 r_{\mathrm{g}}$ results in $v / c=0.08$. Following Meier (2005) the terminal velocity of the steady jet, during the low/hard state, is approximately equal to the escape speed at the footpoint of the magnetic field where the jet is launched, that is at the inner truncated radius of the accretion disk (Meier 2005). The value of $v / c=0.08$ should therefore be comparable with the velocity of the steady jet. Indeed, when LS I +61 303 was essentially quiescent Peracaula et al. (1998) measured an expansion velocity of $0.06 \pm 0.01$. Therefore, on the basis of these consistencies we will assume in the following that the determined value of $300 r_{\mathrm{g}}$ is a good estimate, in terms of order of magnitude, of the truncated radius during the rather low low/hard state of LS I $+61^{\circ} 303$, even if the Ray and Hartman observations of the QPO during a low/hard state of $\mathrm{LS} \mathrm{I}+61^{\circ} 303$ are at another epoch than the MERLIN and VLBA observations of the precessing jet.

In Fig. 4 we show the precessional period (Eq. (4)) due to the Lense-Thirring effect as a function of the dimensionless spin parameter $a_{*}$ for the determined radius $R_{\mathrm{tr}}=300 r_{\mathrm{g}}$. As a result we deduce a period of a few days for the Lense-Thirring precession of a slow rotator $\left(0.001<a_{*}<0.01\right)$. Therefore, Lense-Thirring precession could explain the MERLIN and VLBA observations. However, Eq. (4) assumes a single-particle orbit at the truncation radius $\frac{R_{\mathrm{tr}}}{r_{\mathrm{g}}}=300$, how does that change the result when we consider Lense-Thirring precession of the whole geometrically thick, advection-dominated flow (ADAF) interior up until the truncated disc? An expression of Lense-Thirring precession for disks was first given in Liu \& Melia (2002) and subsequently reproduced in slightly different forms in Fragile et al. (2007) and 


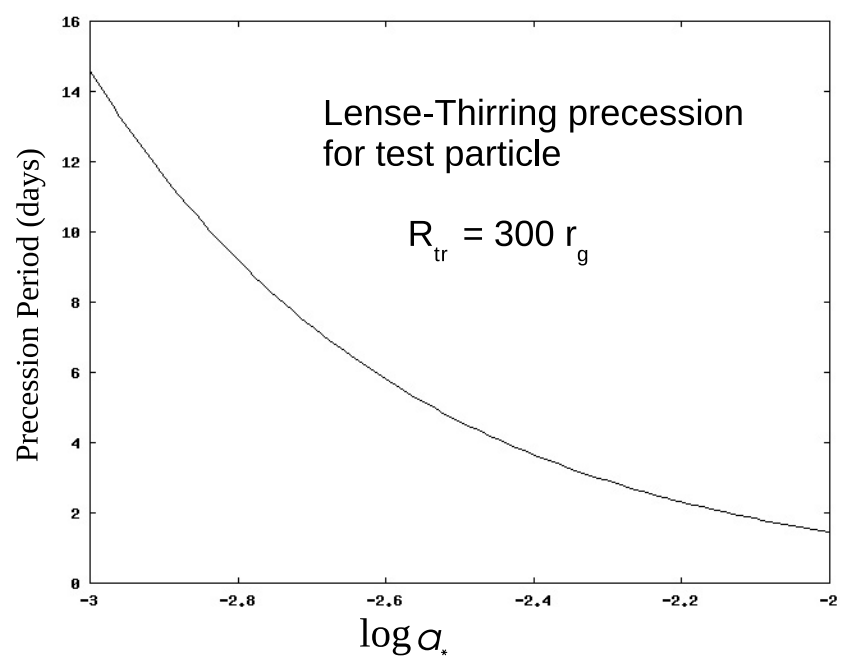

Fig. 4. Lense-Thirring precession for a test particle in function of the dimensionless specific angular momentum, $a_{*}$, (Eq. (4)). The orbit of $R_{\mathrm{tr}}=300 r_{\mathrm{g}}$ has been determined by observed (Ray \& Hartman 2008) $\mathrm{QPO}$ at $2 \mathrm{~Hz}$ during the low/hard state.

Ingram et al. (2009). Equation (2) in Ingram et al. (2009) solved for the precessional period (days) gives:

$P_{\mathrm{LT}}=\frac{1.2 \times 10^{-15}(1+2 \zeta)\left(\frac{R_{\mathrm{tr}}}{r_{\mathrm{g}}}\right)^{(2.5-\zeta)}\left(\frac{r_{\mathrm{i}}}{r_{\mathrm{g}}}\right)^{(0.5+\zeta)}\left(1-{\frac{r_{\mathrm{i}}}{R_{\mathrm{tr}}}}^{(2.5-\zeta)}\right) r_{\mathrm{g}}}{a_{*}(5-2 \zeta)\left(1-{\frac{r_{\mathrm{i}}}{R_{\mathrm{tr}}}}^{(0.5+\zeta)}\right)}$.

The surface density profile through the disk depends on $\zeta$ that for ADAF disk is $\zeta=0.5$ (Ingram et al. 2009). As $r_{\mathrm{i}}$ we have to assume the most inner radius, where the flow remains misaligned and therefore subject to the Lense-Thirring precession, that is $r_{\mathrm{i}}$ must be clearly outside the Bardeen-Petterson effect. As said above Nelson \& Papaloizou (2000) determined the limit of (15-30) $r_{\mathrm{g}}$. However, Nelson \& Papaloizou (2000) used in their study a tilted geometrically thin accretion disk; Fragile \& Anninos (2005) and Fragile et al. (2007) have shown that in case of a geometrically thick disk the Bardeen-Petterson limit is even closer to the compact object and equal to $r_{\mathrm{i}} \sim 10 r_{\mathrm{g}}$. In Fig. 5 we plot Eq. (7) for $r_{\mathrm{i}}=10, R_{\mathrm{tr}}=300$ and $\zeta=0.5$. The result of Fig. 5 is: if the whole ADAF disk is precessing $P_{\mathrm{LT}}$ decreases. In order to mantain a precessional period of some days, as calculated for the single orbit above, it is enough to assume a slower rotating compact object, i.e. with lower $a_{*}$.

\section{Conclusions}

Two consecutive MERLIN observations of LS I $+61^{\circ} 303$ showed a rotation of the position angle of the radio structure of $\simeq 60^{\circ}$ in only $24 \mathrm{~h}$ (Massi et al. 2004). Several consecutive VLBA images by Dhawan et al. (2006), three days apart, have confirmed the fast variations. In this paper we analyse precession due to the tidal torque induced by the Be star and to the Lense-Thirring precession induced by the tilted rotating compact object.

It is unlikely that the observed days time scale could be created by tidal precession. In this paper we show that this mechanism would produce too large a precessional period of $P_{\mathrm{TF}} \geq 460 \mathrm{~d}$. To lower the precessional period to a few days one should increase the disk size above the limit of any stable disk.

On the contrary, for Lense-Thirring precession for a singleparticle orbit we determine that a slow rotator, with $0.001<$ $a_{*}<0.01$, induces a $P_{\mathrm{LT}}$ of a few days for a truncated radius

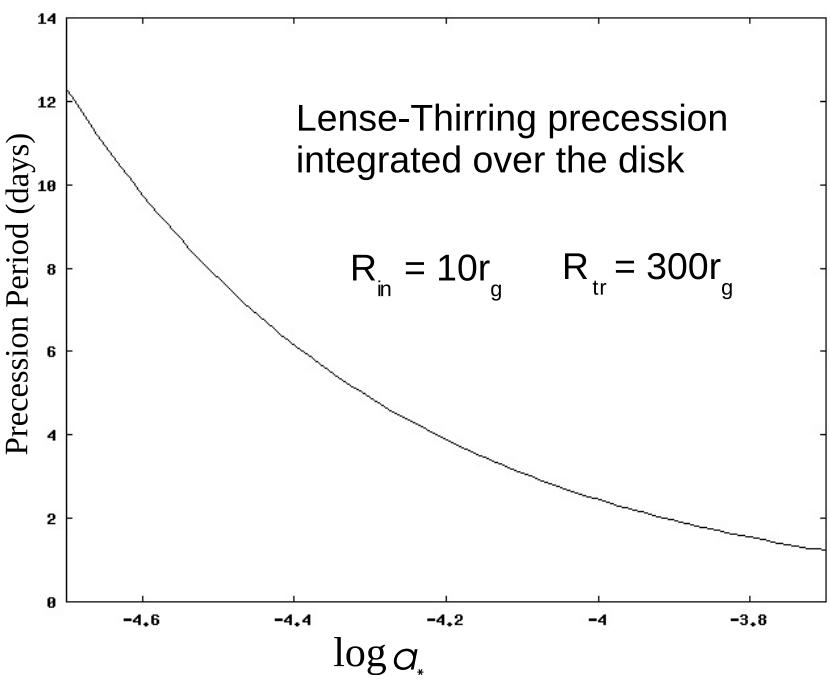

Fig. 5. Lense-Thirring precession for a geometrically thick accretion flow with inner and outer radii $r_{\mathrm{i}}$ and $R_{\mathrm{tr}}$ as in Eq. (7) for $\zeta=0.5$.

$R_{\mathrm{tr}}=300 r_{\mathrm{g}}$. The large truncated radius, $R_{\mathrm{tr}}$, that we derived from QPO observed with RXTE (Ray \& Hartman 2008), is consistent with the low/hard state of LS I $+61^{\circ} 303$ during those observations (spectrum best fit by a powerlaw of photon index about 1.5; Ray \& Hartman 2008). Moreover, the escape velocity, that following Meier (2005) is equal to the velocity of the steady jet, for $R_{\mathrm{tr}}=300 r_{\mathrm{g}}$ results in $v / c=0.08$ and is therefore consistent with the expansion velocity of $0.06 \pm 0.01$ observed by Peracaula et al. (1998) in an epoch when LS I +61 303 was essentially quiescent.

Taking into account the precession of the whole hot, inner thick ADAF disk (between the Bardeen-Petterson limiting radius of $10 r_{\mathrm{g}}$ and the truncated radius of $300 r_{\mathrm{g}}$ ) we obtain an even smaller $P_{\mathrm{LT}}$. In order to mantain a precessional period of some days, as calculated for the single orbit, it is enough to assume a slower rotating compact object, i.e. with lower $a_{*}$. We conclude that the Lense-Thirring mechanism can be applied to LS I $+61^{\circ} 303$ and so explain the observed fast variations.

Acknowledgements. We are grateful to the anonymous referee for the constructive comments that improved the paper and to Marina Kaufman Bernadó and Johannes Schmid-Burgk for comments and suggestions. The work of L. Zimmermann is partly supported by the German Excellence Initiative via the Bonn Cologne Graduate School.

\section{References}

Abdo, A. A., Ackermann, M., Ajello, M., et al. 2009, ApJ, 701, L123

Aharonian, F., Akhperjanian, A. G., Anton, G., et al. 2009, A\&A, 507, 389 Acciari, V. A., Aliu, E., Arlen, T., et al. 2009, ApJ, 700, 1034

Albert, J., Aliu, E., Anderhub, H., et al. 2006, Science, 312, 1771

Albert, J., Aliu, E., Anderhub, H., et al. 2009, ApJ, 693, 303

Anderhub, H. 2009 ApJ, 706, L27

Aragona, C., McSwain, M. V., Grundstrom, E. D., et al. 2009, ApJ, 698, 514 Bondi, H. 1952, MNRAS, 112, 195B

Bosch-Ramon, V., Paredes, J. M., Romero, G. E., \& Ribó, M. 2006, A\&A, 459, L25

Casares, J., Ribas, I., Paredes, J. M., Martí, J., \& Allende Prieto, C. 2005, MNRAS, 360, 1105

Chernyakova, M., Neronov, A., \& Walter, R. 2006, MNRAS, 372, 1585

Collin, S. \& Huré, J.-M. 1999, A\&A, 341, 385

Caproni, A., Livio, M., Abraham, Z., \& Mosquera Cuesta, H. J. 2006, ApJ, 653, 112

Connors, T. W., Johnston, S., Manchester, R. N., \& McConnell, D. 2002, MNRAS, 336, 1201 
Dhawan, V., Mioduszewski, A., \& Rupen, M. 2006, Proceedings of the VI Microquasar Workshop, 52

Dubner, G. M., Holdaway, M., Goss, W. M., \& Mirabel, I. F. 1998, AJ, 116, 1842

Fender, R. P., Belloni, T. M., \& Gallo, E. 2004, MNRAS, 355, 1105

Fragile, P. C., \& Anninos, P. 2005, ApJ, 623, 347

Fragile, P. C., Mathews, G. J., \& Wilson, J. R. 2001, ApJ, 553, 955

Fragile, P. C., Blaes, O. M., Anninos, P., \& Salmonson, J. D. 2007, ApJ, 668, 417

Foulkes, S. B., Haswell, C. A., \& Murray, J. R. 2006, MNRAS, 366, 1399

Gregory, P. C. 2002, ApJ, 575, 427

Hutchings, J. B., \& Crampton, D. 1981, PASP, 93, 486

Ingram, A., \& Done, C. 2010, MNRAS, accepted [arXiv: 0907. 5485]

Ingram, A., Done, C., \& Fragile, P. 2009, MNRAS, 397, L101

Johnston, S., Lyne, A. G., Manchester, R. N., et al. 1992, MNRAS, 255, 401

Kaufman Bernadó, M. M., Romero, G. E., \& Mirabel, I. F. 2002, A\&A, 385, L10

Katarzyǹski, K., \& Walczewska, K. 2010, A\&A, 510, A63

Katarzyński, K., Ghisellini, G., Tavecchio, F., et al. 2005, A\&A, 433, 479

Larwood, J. 1998, MNRAS, 299, L32

Liu, S., \& Melia, F. 2002, ApJ, 573, L23

Marscher, A. P., \& Gear, W. K. 1985, ApJ, 298, 114

Martí, J., \& Paredes, J. M. 1995, A\&A, 298, 151

Massi, M., Ribó, M, Paredes, J. M., et al. 2004, A\&A, 414, L1
Massi, M., Ribó, M., Paredes, J. M., et al. 2005, High Energy Gamma-Ray Astronomy, 745, 311

Massi, M., \& Kaufman Bernadó, M. 2009, ApJ, 702, 1179

McClintock, J. E., \& Remillard, R. A. 2006, Compact stellar X-ray sources (Cambridge University Press), 157

Meier, D. L. 2005, Ap\&SS, 300, 55

Nelson, R. P., \& Papaloizou, J. C. B. 2000, MNRAS, 315, 570

Paczyǹski, B. 1977, ApJ, 216, 822

Papaloizou, J. C. B., \& Pringle, J. E. 1977, MNRAS, 181, 441

Peracaula, M., Gabuzda, D. C., \& Taylor, A. R. 1998, A\&A, 330, 612

Ray, P. S., Jacob, M., \& Hartman, J. M. 2008, The Astronomer's Telegram, 1730, 1

Stella, L., \& Vietri, M. 1998, ApJ, 492, L59

Taylor, A. R., Kenny, H. T., Spencer, R. E., \& Tzioumis, A. 1992, ApJ, 395, 268

Taylor, A. R., Dougherty, S. M., Scott, W. K., Peracaula, M., \& Paredes, J. M. 2000, proc. of Astrophysical Phenomena Revealed by Space VLBI, ed. H. Hirabayashi, P. G. Edwards, \& D. W. Murphy, Pub. ISAS, 223

Waters, L. B. F. M., Taylor, A. R., van den Heuvel, E. P. J., Habets, G. M. H. J., \& Persi, P. 1988, A\&A, 198, 200

Wilkins, D. C. 1972, Phys. Rev. D, 5, 814

Zamanov, R. K., \& Martí, J. 2000, A\&A, 358, L55 